\title{
Determining the Relative Reactivity of Sulfate, Bisulfate and Organosulfates with Epoxides on Secondary Organic Aerosol
}

\author{
Erika Aoki, Jon N. Sarrimanolis, Sophie A. Lyon and Matthew J. Elrod* \\ Department of Chemistry and Biochemistry, Oberlin College, Oberlin, Ohio, 44074 USA \\ Email: mjelrod@oberlin.edu
}

Contents: NMR assignments, solution parameters, observed sulfate product yields

(14 pages, 5 tables, 11 figures)

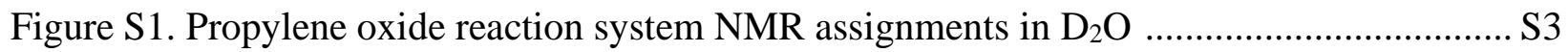

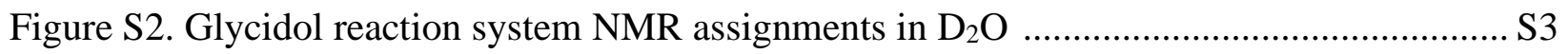

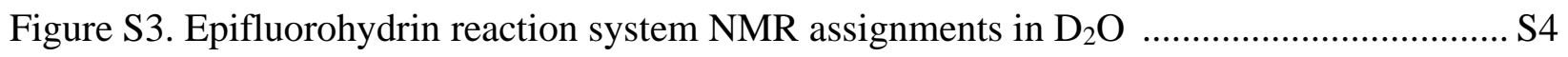

Figure S4. Epifluorohydrin reaction system NMR assignments in $\mathrm{D}_{2} \mathrm{O} \quad$............................... S4

Figure S5. Epibromohydrin reaction system NMR assignments in $\mathrm{D}_{2} \mathrm{O}$.............................. S5

Figure S6. Cis-2,3-epoxybutane reaction system NMR assignments in $\mathrm{D}_{2} \mathrm{O}$......................... S5

Figure S7. Cis-2,3-epoxy-1,4-butanediol reaction system NMR assignments in $\mathrm{D}_{2} \mathrm{O}$.............. S6

Figure S8. 1,2-epoxybutane reaction system NMR assignments in $\mathrm{D}_{2} \mathrm{O}$............................. S6

Figure S9. 2-methyl-1,2-epoxypropane reaction system NMR assignments in $\mathrm{D}_{2} \mathrm{O}$............... S7

Figure S10 2,3-dimethyl-2,3-epoxybutane reaction system NMR assignments in $\mathrm{D}_{2} \mathrm{O}$........... S7

Figure S11. Trans- $\beta$-IEPOX reaction system NMR assignments in $\mathrm{D}_{2} \mathrm{O}$............................ S8

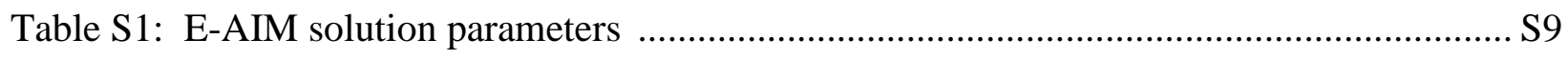

Table S2: Observed sulfate percentage product yields and water activity weighted mole fraction ratios for 1,2-epoxybutane S10

Table S3: Observed sulfate percentage product yields and water activity weighted mole fraction ratios for cis-2,3-epoxybutane 
Table S4: Observed sulfate percentage product yields and water activity weighted mole fraction

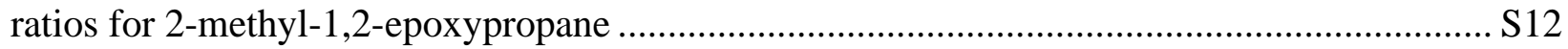

Table S5: Observed sulfate and methylsulfate mole fraction yields.......................................... S13

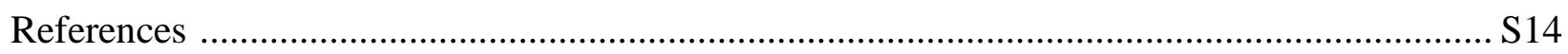




\section{NMR Assignments}

NMR assignments are given for each system in the following sections, along with NMR referencing information. Species are primarily assigned using ${ }^{13} \mathrm{C}$ NMR, as ${ }^{1} \mathrm{H}$ NMR spectra were often uninterpretable due to chemical shift overlap between the various nucleophilic addition products.
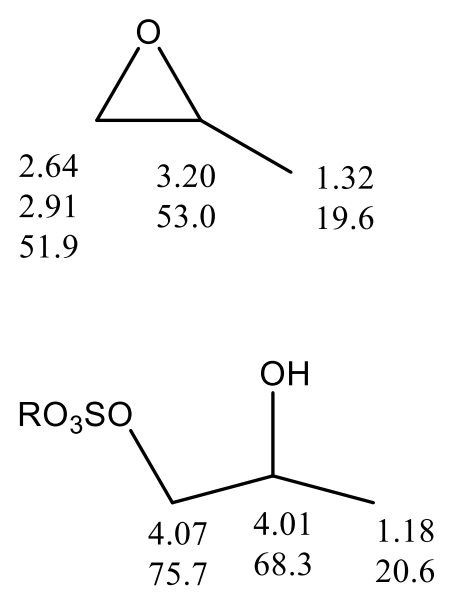
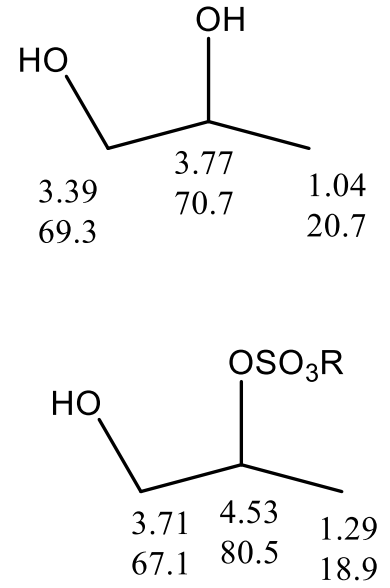

Figure S1. Propylene oxide reaction system NMR assignments in $\mathbf{D}_{2} \mathbf{O},{ }^{1} \mathrm{H}$ referenced to $\mathrm{HDO}$ at $4.79 \mathrm{ppm}$ and ${ }^{13} \mathrm{C}$ referenced to secondary calibration of primary 1,2-butanediol carbon atom at $69.3 \mathrm{ppm}$ (primary calibration is to DSS at $0.0 \mathrm{ppm}$ ).
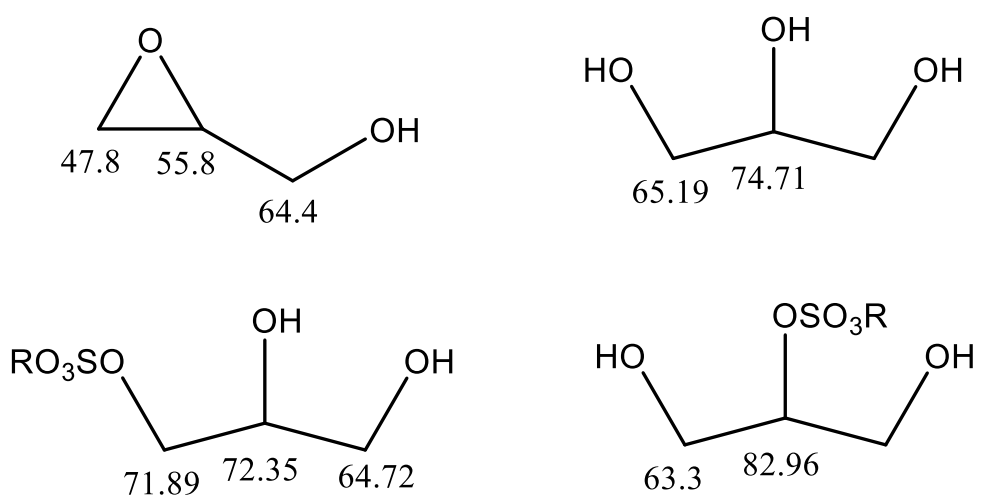

Figure S2. Glycidol reaction system NMR assignments in $\mathbf{D}_{2} \mathbf{O},{ }^{13} \mathrm{C}$ referenced to DSS at 0.0 ppm. 
Due to presence of the spin $1 / 2{ }^{19} \mathrm{~F}$ nucleus, each ${ }^{1} \mathrm{H}$ and ${ }^{13} \mathrm{C}$ peak is split into a doublet (or more for ${ }^{1} \mathrm{H}$ spectra). The center chemical shifts and coupling constants for each type of coupling interaction are given below.
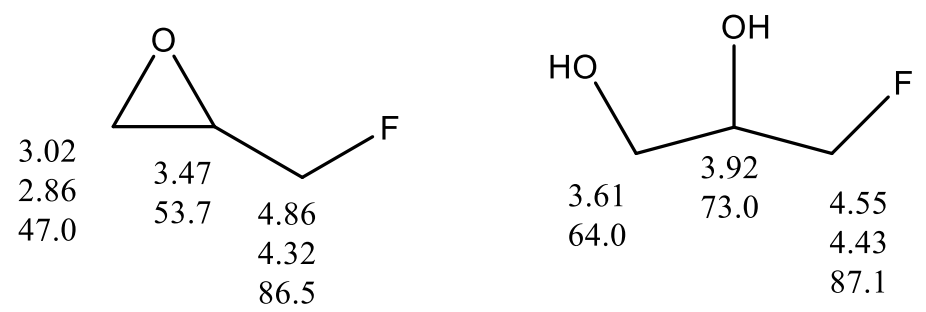

$$
\begin{aligned}
& \mathrm{J}\left({ }^{1} \mathrm{H}-{ }^{19} \mathrm{~F}\right) \text { ipso: } 0.12 \mathrm{ppm} \\
& \mathrm{J}\left({ }^{13} \mathrm{C}-{ }^{19} \mathrm{~F}\right) \text { ipso: } 1.7 \mathrm{ppm} \\
& \mathrm{J}\left({ }^{13} \mathrm{C}-{ }^{19} \mathrm{~F}\right) \text { alpha: } 0.3 \mathrm{ppm} \\
& \mathrm{J}\left({ }^{13} \mathrm{C}-{ }^{19} \mathrm{~F}\right) \text { beta: } 0.1 \mathrm{ppm}
\end{aligned}
$$
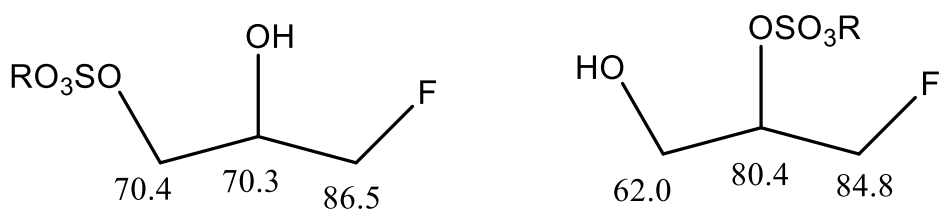

Figure S3. Epifluorohydrin reaction system NMR assignments in $\mathbf{D}_{2} \mathbf{O}$, both ${ }^{1} \mathrm{H}$ and ${ }^{13} \mathrm{C}$ referenced to DSS at $0.0 \mathrm{ppm}$.
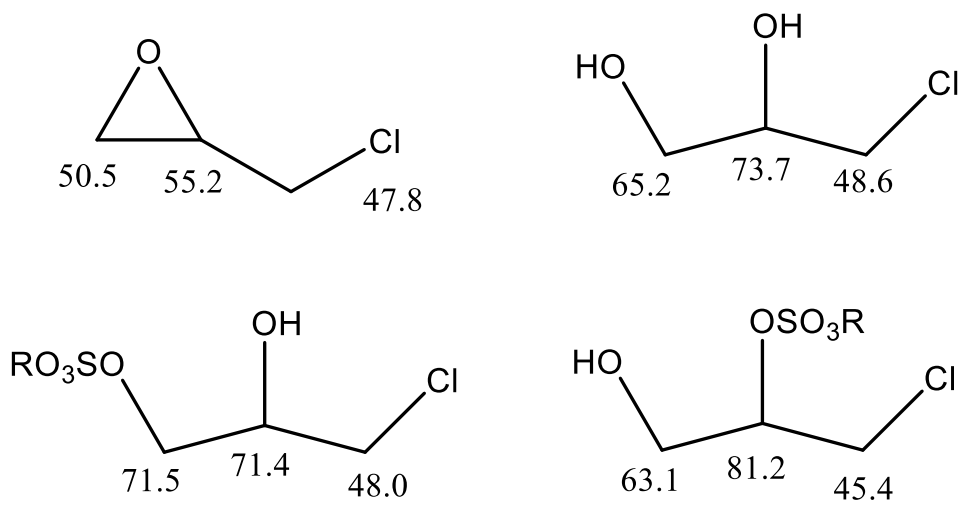

Figure S4. Epifluorohydrin reaction system NMR assignments in $\mathbf{D}_{2} \mathbf{O},{ }^{13} \mathrm{C}$ referenced to DSS at $0.0 \mathrm{ppm}$ 

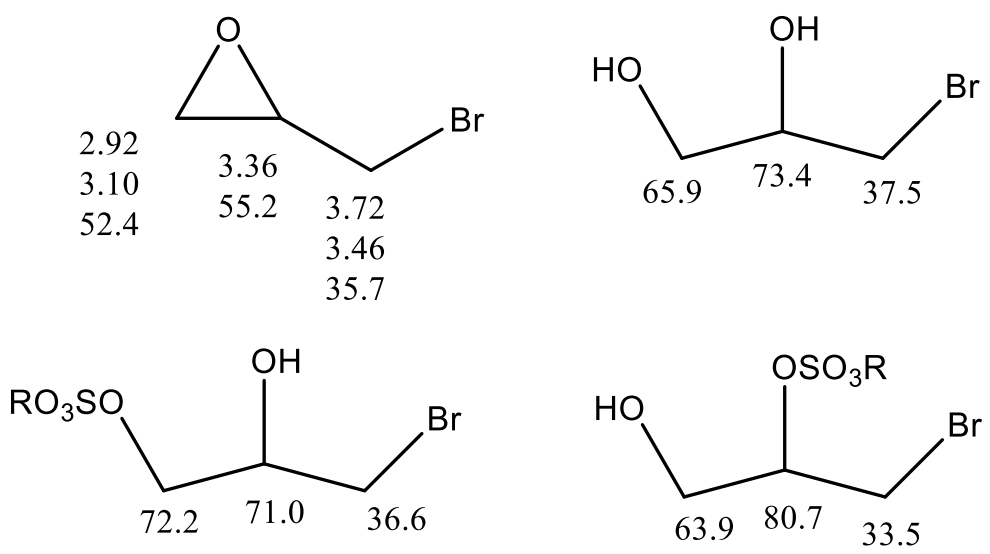

Figure S5. Epibromohydrin reaction system NMR assignments in $\mathbf{D}_{2} \mathbf{O},{ }^{1} \mathrm{H}$ referenced to $\mathrm{HDO}$ at $4.79 \mathrm{ppm}$ and ${ }^{13} \mathrm{C}$ referenced to secondary calibration of primary 3-bromo-1,2butanediol carbon atom at $65.9 \mathrm{ppm}$ (primary calibration is to DSS at $0.0 \mathrm{ppm}$ ).
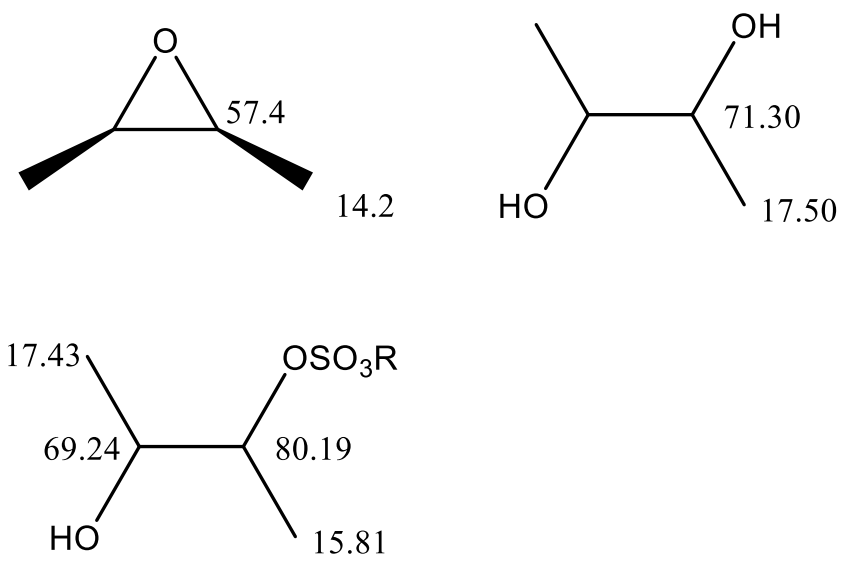

Figure S6. Cis-2,3-epoxybutane reaction system NMR assignments in $\mathbf{D}_{2} \mathbf{O},{ }^{13} \mathrm{C}$ referenced to DSS at $0.0 \mathrm{ppm}$. 

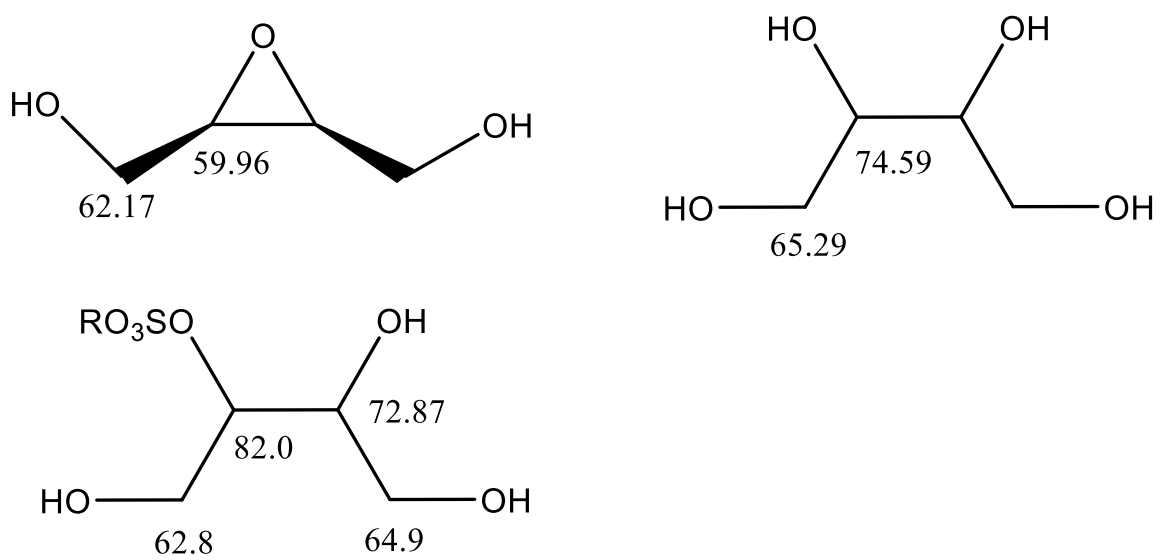

Figure S7. Cis-2,3-epoxy-1,4-butanediol reaction system NMR assignments in $\mathbf{D}_{2} \mathrm{O},{ }^{13} \mathrm{C}$ referenced to DSS at $0.0 \mathrm{ppm}$.

$2.90,2.69$

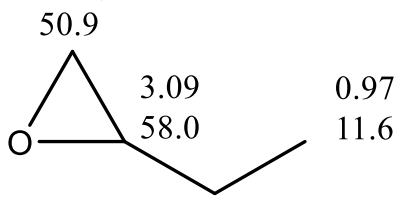

$1.63,1.50$

27.3

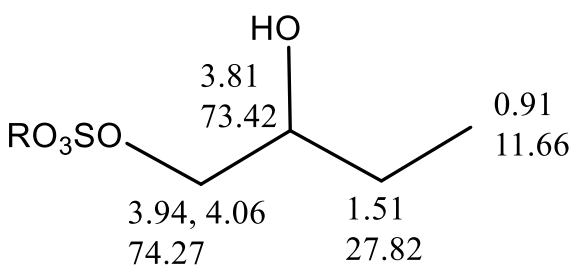

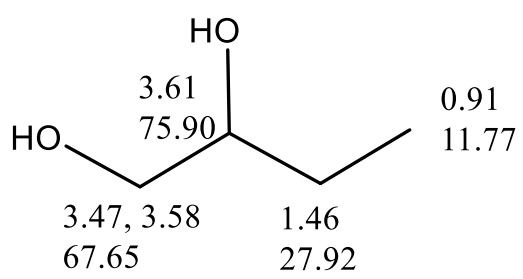

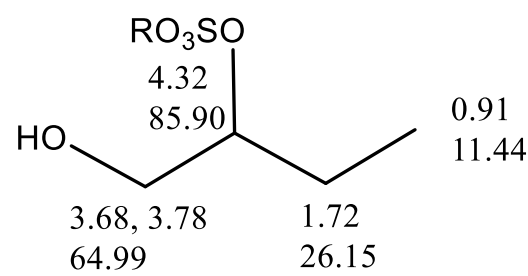

Figure S8. 1,2-epoxybutane reaction system NMR assignments in $\mathrm{D}_{2} \mathrm{O}$. both ${ }^{1} \mathrm{H}$ and ${ }^{13} \mathrm{C}$ referenced to DSS at $0.0 \mathrm{ppm}$. 

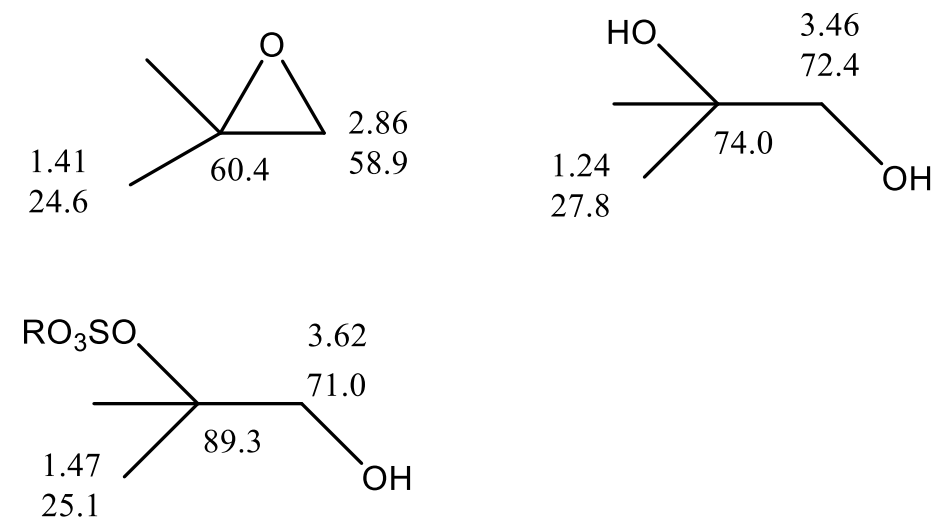

Figure S9. 2-methyl-1,2-epoxypropane reaction system NMR assignments in $\mathrm{D}_{2} \mathrm{O}$. both ${ }^{1} \mathrm{H}$ and ${ }^{13} \mathrm{C}$ referenced to DSS at $0.0 \mathrm{ppm}$.
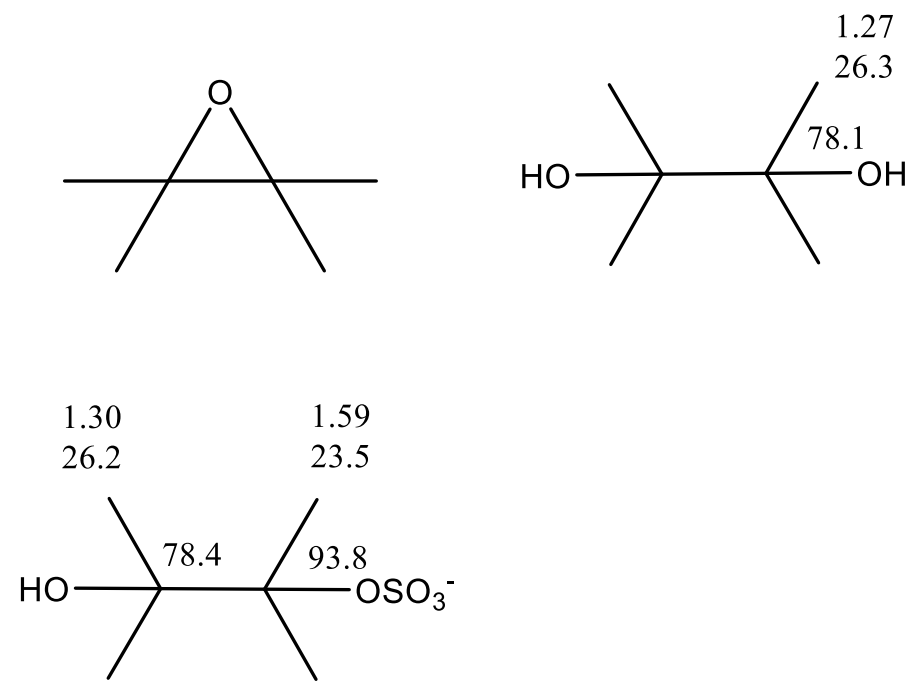

Figure S10 2,3-dimethyl-2,3-epoxybutane reaction system NMR assignments in $\mathrm{D}_{2} \mathrm{O}$. both ${ }^{1} \mathrm{H}$ and ${ }^{13} \mathrm{C}$ referenced to DSS at $0.0 \mathrm{ppm}$. 

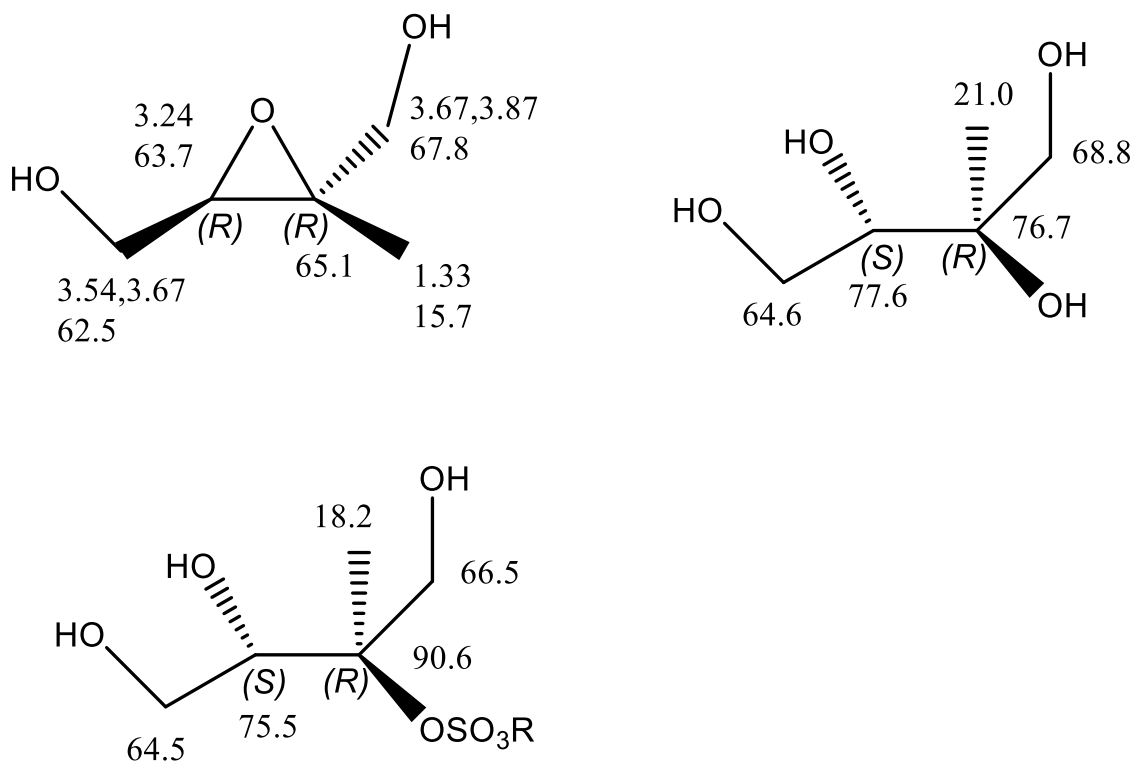

Figure S11. Trans- $\beta$-IEPOX reaction system NMR assignments in $\mathbf{D}_{2} \mathbf{O}$. both ${ }^{1} \mathrm{H}$ and ${ }^{13} \mathrm{C}$ referenced to DSS at $0.0 \mathrm{ppm}$. 


\begin{tabular}{|c|c|c|c|c|c|c|c|c|c|c|c|c|c|c|c|}
\hline & {$\left[\mathrm{SO}_{4}{ }^{2-}\right](\mathrm{m})$} & $\mathrm{XSO}_{4}{ }^{2-}$ & $\gamma \mathrm{SO}_{4}^{2-}$ & a $\mathrm{SO}_{4}{ }^{2-}(\mathrm{m})$ & {$\left[\mathrm{HSO}_{4}{ }^{-}\right](\mathrm{m})$} & $\mathrm{X} \mathrm{HSO}_{4}^{-}$ & $\gamma \mathrm{HSO}_{4}^{-}$ & $\mathrm{a} \mathrm{HSO}_{4}^{-}(\mathrm{m})$ & $\mathrm{X} \mathrm{H}_{2} \mathrm{O}$ & $\gamma \mathrm{H} 2 \mathrm{O}$ & a $\mathrm{H} 2 \mathrm{O}(\mathrm{m})$ & {$\left[\mathrm{H}^{+}\right](\mathrm{m})$} & $\mathrm{XH}^{+}$ & $\gamma \mathrm{H}+$ & a $\mathrm{H}+(\mathrm{m})$ \\
\hline \multicolumn{16}{|l|}{ Solution Set 1} \\
\hline $0.1 \mathrm{M} \mathrm{H}_{2} \mathrm{SO}_{4}$ & 0.02685 & 0.00048 & 0.23490 & 0.00631 & 0.07315 & 0.00131 & 0.78660 & 0.05754 & 0.99590 & 1.00000 & 55.50000 & 0.12690 & 0.00228 & 0.75810 & 0.09620 \\
\hline $0.1 \mathrm{M} \mathrm{H}_{2} \mathrm{SO}_{4} 0.1 \mathrm{M} \mathrm{Na}_{2} \mathrm{SO}_{4}$ & 0.09153 & 0.00164 & 0.15030 & 0.01376 & 0.10847 & 0.00194 & 0.72390 & 0.07852 & 0.99120 & 1.00100 & 55.55550 & 0.09153 & 0.00164 & 0.66070 & 0.06047 \\
\hline $0.1 \mathrm{M} \mathrm{H}_{2} \mathrm{SO}_{4} 0.2 \mathrm{M} \mathrm{Na}_{2} \mathrm{SO}_{4}$ & 0.17700 & 0.00314 & 0.11070 & 0.01959 & 0.12300 & 0.00219 & 0.67800 & 0.08339 & 0.98620 & 1.00300 & 55.66650 & 0.07695 & 0.00137 & 0.58910 & 0.04533 \\
\hline $0.1 \mathrm{M} \mathrm{H}_{2} \mathrm{SO}_{4} 0.3 \mathrm{M} \mathrm{Na}_{2} \mathrm{SO}_{4}$ & 0.27000 & 0.00477 & 0.08805 & 0.02377 & 0.12990 & 0.00230 & 0.64360 & 0.08360 & 0.98110 & 1.00400 & 55.72200 & 0.07001 & 0.00124 & 0.53780 & 0.03765 \\
\hline $0.1 \mathrm{M} \mathrm{H}_{2} \mathrm{SO}_{4} 0.4 \mathrm{M} \mathrm{Na}_{2} \mathrm{SO}_{4}$ & 0.36610 & 0.00644 & 0.07322 & 0.02681 & 0.13390 & 0.00235 & 0.61640 & 0.08254 & 0.97600 & 1.00600 & 55.83300 & 0.06615 & 0.00116 & 0.50050 & 0.03311 \\
\hline $0.1 \mathrm{M} \mathrm{H}_{2} \mathrm{SO}_{4} 0.5 \mathrm{M} \mathrm{Na}_{2} \mathrm{SO}_{4}$ & 0.46370 & 0.00811 & 0.06270 & 0.02907 & 0.13627 & 0.00238 & 0.59410 & 0.08096 & 0.97090 & 1.00080 & 55.54440 & 0.06373 & 0.00112 & 0.47240 & 0.03011 \\
\hline $0.1 \mathrm{M} \mathrm{H}_{2} \mathrm{SO}_{4} 0.7 \mathrm{M} \mathrm{Na}_{2} \mathrm{SO}_{4}$ & 0.66080 & 0.01144 & 0.04868 & 0.03217 & 0.13920 & 0.00241 & 0.55930 & 0.07785 & 0.96090 & 1.01200 & 56.16600 & 0.60819 & 0.00105 & 0.43480 & 0.26444 \\
\hline $0.1 \mathrm{M} \mathrm{H}_{2} \mathrm{SO}_{4} 0.9 \mathrm{M} \mathrm{Na}_{2} \mathrm{SO}_{4}$ & 0.85880 & 0.01472 & 0.03975 & 0.03414 & 0.14112 & 0.00242 & 0.53080 & 0.07491 & 0.95100 & 1.01600 & 56.38800 & 0.58830 & 0.00101 & 0.41320 & 0.24309 \\
\hline & & & & & & & & & & & & & & & \\
\hline \multicolumn{16}{|l|}{ Solution Set 2} \\
\hline $0.1 \mathrm{M} \mathrm{NaHSO}_{4}$ & 0.04989 & 0.00089 & 0.22540 & 0.01125 & 0.05011 & 0.00090 & 0.75050 & 0.03761 & 0.99550 & 1.00100 & 55.55550 & 0.04989 & 0.00089 & 0.70690 & 0.03527 \\
\hline $0.2 \mathrm{M} \mathrm{NaHSO}_{4}$ & 0.09153 & 0.00164 & 0.15030 & 0.01376 & 0.10847 & 0.00194 & 0.72390 & 0.07852 & 0.99120 & 1.00100 & 55.55550 & 0.09153 & 0.00164 & 0.66070 & 0.06047 \\
\hline $0.3 \mathrm{M} \mathrm{NaHSO}_{4}$ & 0.13257 & 0.00236 & 0.11340 & 0.01503 & 0.16743 & 0.00298 & 0.71250 & 0.11929 & 0.98700 & 1.00200 & 55.61100 & 0.13257 & 0.00236 & 0.63670 & 0.08441 \\
\hline $0.4 \mathrm{M} \mathrm{NaHSO}_{4}$ & 0.17380 & 0.00308 & 0.09086 & 0.01579 & 0.22620 & 0.00401 & 0.70720 & 0.15997 & 0.98280 & 1.00300 & 55.66650 & 0.17380 & 0.00308 & 0.62270 & 0.10823 \\
\hline $0.5 \mathrm{M} \mathrm{NaHSO}_{4}$ & 0.21545 & 0.00380 & 0.07551 & 0.01627 & 0.28455 & 0.00502 & 0.70550 & 0.20075 & 0.97860 & 1.00400 & 55.72200 & 0.21545 & 0.00380 & 0.61460 & 0.13242 \\
\hline $0.6 \mathrm{M} \mathrm{NaHSO}_{4}$ & 0.25750 & 0.00452 & 0.06436 & 0.01657 & 0.34250 & 0.00601 & 0.70600 & 0.24181 & 0.97440 & 1.00500 & 55.77750 & 0.25720 & 0.00452 & 0.61050 & 0.15702 \\
\hline $0.7 \mathrm{M} \mathrm{NaHSO}_{4}$ & 0.29998 & 0.00524 & 0.05590 & 0.01677 & 0.40002 & 0.00699 & 0.70830 & 0.28333 & 0.97030 & 1.00600 & 55.83300 & 0.29998 & 0.00524 & 0.60950 & 0.18284 \\
\hline $0.8 \mathrm{M} \mathrm{NaHSO}_{4}$ & 0.34270 & 0.00597 & 0.04928 & 0.01689 & 0.45720 & 0.00796 & 0.71190 & 0.32548 & 0.96620 & 1.00600 & 55.83300 & 0.34270 & 0.00597 & 0.61100 & 0.20939 \\
\hline $0.9 \mathrm{M} \mathrm{NaHSO}_{4}$ & 0.38575 & 0.00669 & 0.04397 & 0.01696 & 0.51425 & 0.00891 & 0.71670 & 0.36856 & 0.96210 & 1.00700 & 55.88850 & 0.38575 & 0.00669 & 0.61470 & 0.23712 \\
\hline $1.0 \mathrm{M} \mathrm{NaHSO}_{4}$ & 0.42887 & 0.00740 & 0.03963 & 0.01700 & 0.57110 & 0.00986 & 0.72240 & 0.41256 & 0.95810 & 1.00800 & 55.94400 & 0.42880 & 0.00740 & 0.62030 & 0.26598 \\
\hline $1.5 \mathrm{M} \mathrm{NaHSO}_{4}$ & 0.64280 & 0.01087 & 0.02626 & 0.01688 & 0.85720 & 0.01449 & 0.76160 & 0.65284 & 0.93840 & 1.01000 & 56.05500 & 0.64280 & 0.01087 & 0.67310 & 0.43267 \\
\hline $2.0 \mathrm{M} \mathrm{NaHSO}_{4}$ & 0.84543 & 0.01401 & 0.01960 & 0.01657 & 1.15460 & 0.01913 & 0.81290 & 0.93857 & 0.91970 & 1.01000 & 56.05500 & 0.84543 & 0.01401 & 0.76470 & 0.64650 \\
\hline $3.0 \mathrm{M} \mathrm{NaHSO}_{4}$ & 1.18500 & 0.01890 & 0.01325 & 0.01570 & 1.81500 & 0.02895 & 0.93220 & 1.69194 & 0.88540 & 1.00400 & 55.72200 & 1.18500 & 0.01890 & 1.07800 & 1.27743 \\
\hline $4.0 \mathrm{M} \mathrm{NaHSO}_{4}$ & 1.42000 & 0.02187 & 0.01022 & 0.01451 & 2.58000 & 0.03973 & 1.05200 & 2.71416 & 0.85490 & 0.99020 & 54.95610 & 1.42000 & 0.02187 & 1.61800 & 2.29756 \\
\hline $5.0 \mathrm{M} \mathrm{NaHSO}_{4}$ & 1.56100 & 0.02328 & 0.00838 & 0.01308 & 3.43900 & 0.05127 & 1.15800 & 3.98236 & 0.82760 & 0.97230 & 53.96265 & 1.56100 & 0.02328 & 2.47400 & 3.86191 \\
\hline
\end{tabular}

\section{Table S1: E-AIM ${ }^{1}$ solution parameters}




\begin{tabular}{|c|c|c|c|c|c|c|}
\hline & \multicolumn{2}{|r|}{ 1-sulfato } & \multicolumn{2}{|r|}{ 2-sulfato } & \multicolumn{2}{|r|}{ total } \\
\hline & $\%$ yield & $\left(Y_{\text {sulfate }} / Y_{\text {water }}\right) * a_{\text {water }}$ & $\%$ yield & $\left(Y_{\text {sulfate }} / Y_{\text {water }}\right) * a_{\text {water }}$ & $\%$ yield & $\left(Y_{\text {sulfate }} / Y_{\text {water }}\right) * a_{\text {water }}$ \\
\hline \multicolumn{7}{|l|}{ Solution Set 1} \\
\hline $0.1 \mathrm{M} \mathrm{D}_{2} \mathrm{SO}_{4} 0.1 \mathrm{M} \mathrm{Na}_{2} \mathrm{SO}_{4}$ & 4.73 & 2.85 & 3.08 & 1.86 & 7.82 & 4.71 \\
\hline $0.1 \mathrm{M} \mathrm{D}_{2} \mathrm{SO}_{4} 0.3 \mathrm{M} \mathrm{Na}_{2} \mathrm{SO}_{4}$ & 7.76 & 5.04 & 6.45 & 4.19 & 14.21 & 9.23 \\
\hline $0.1 \mathrm{M} \mathrm{D}_{2} \mathrm{SO}_{4} 0.5 \mathrm{M} \mathrm{Na}_{2} \mathrm{SO}_{4}$ & 9.74 & 6.62 & 8.49 & 5.77 & 18.23 & 12.38 \\
\hline $0.1 \mathrm{M} \mathrm{D}_{2} \mathrm{SO}_{4} 0.7 \mathrm{M} \mathrm{Na}_{2} \mathrm{SO}_{4}$ & 11.38 & 8.09 & 9.61 & 6.83 & 20.99 & 14.92 \\
\hline $0.1 \mathrm{M} \mathrm{D}_{2} \mathrm{SO}_{4} 0.9 \mathrm{M} \mathrm{Na}_{2} \mathrm{SO}_{4}$ & 11.35 & 8.12 & 9.80 & 7.01 & 21.15 & 15.13 \\
\hline $0.1 \mathrm{M} \mathrm{D}_{2} \mathrm{SO}_{4} 0.1 \mathrm{M} \mathrm{Na}_{2} \mathrm{SO}_{4}$ & 4.06 & 2.47 & 4.63 & 2.82 & 8.70 & 5.29 \\
\hline $0.1 \mathrm{M} \mathrm{D}_{2} \mathrm{SO}_{4} 0.3 \mathrm{M} \mathrm{Na}_{2} \mathrm{SO}_{4}$ & 7.25 & 4.71 & 6.96 & 4.52 & 14.21 & 9.23 \\
\hline $0.1 \mathrm{M} \mathrm{D}_{2} \mathrm{SO}_{4} 0.5 \mathrm{M} \mathrm{Na}_{2} \mathrm{SO}_{4}$ & 9.20 & 6.24 & 8.93 & 6.06 & 18.13 & 12.30 \\
\hline $0.1 \mathrm{M} \mathrm{D}_{2} \mathrm{SO}_{4} 0.7 \mathrm{M} \mathrm{Na}_{2} \mathrm{SO}_{4}$ & 12.05 & 8.85 & 11.45 & 8.40 & 23.49 & 17.25 \\
\hline $0.1 \mathrm{M} \mathrm{D}_{2} \mathrm{SO}_{4} 0.9 \mathrm{M} \mathrm{Na}_{2} \mathrm{SO}_{4}$ & 11.15 & 8.09 & 11.15 & 8.09 & 22.30 & 16.19 \\
\hline \multicolumn{7}{|l|}{ Solution Set 2} \\
\hline $0.1 \mathrm{M} \mathrm{NaDSO}_{4}$ & 2.41 & 1.42 & 2.94 & 1.72 & 5.35 & 3.14 \\
\hline $0.2 \mathrm{M} \mathrm{NaDSO}_{4}$ & 2.08 & 1.22 & 3.40 & 2.00 & 5.48 & 3.22 \\
\hline $0.3 \mathrm{M} \mathrm{NaDSO}_{4}$ & 3.38 & 2.02 & 3.69 & 2.21 & 7.07 & 4.23 \\
\hline $0.4 \mathrm{M} \mathrm{NaDSO}_{4}$ & 4.50 & 2.73 & 3.74 & 2.27 & 8.24 & 5.00 \\
\hline $0.5 \mathrm{M} \mathrm{NaDSO}_{4}$ & 4.67 & 2.85 & 4.00 & 2.44 & 8.67 & 5.29 \\
\hline $0.6 \mathrm{M} \mathrm{NaDSO}_{4}$ & 5.14 & 3.16 & 4.15 & 2.55 & 9.29 & 5.71 \\
\hline $0.7 \mathrm{M} \mathrm{NaDSO}_{4}$ & 5.17 & 3.20 & 4.52 & 2.79 & 9.69 & 5.99 \\
\hline $0.8 \mathrm{M} \mathrm{NaDSO}_{4}$ & 5.33 & 3.30 & 4.65 & 2.88 & 9.98 & 6.19 \\
\hline $0.9 \mathrm{M} \mathrm{NaDSO}_{4}$ & 6.51 & 4.09 & 4.68 & 2.94 & 11.18 & 7.04 \\
\hline $1.0 \mathrm{M} \mathrm{NaDSO}_{4}$ & 5.94 & 3.73 & 5.03 & 3.16 & 10.98 & 6.90 \\
\hline $1.5 \mathrm{M} \mathrm{NaDSO}_{4}$ & 7.31 & 4.68 & 5.19 & 3.32 & 12.50 & 8.01 \\
\hline $2.0 \mathrm{M} \mathrm{NaDSO}_{4}$ & 8.31 & 5.41 & 5.57 & 3.62 & 13.88 & 9.03 \\
\hline $3.0 \mathrm{M} \mathrm{NaDSO}_{4}$ & 11.30 & 7.68 & 6.78 & 4.61 & 18.07 & 12.29 \\
\hline $4.0 \mathrm{M} \mathrm{NaDSO}_{4}$ & 13.02 & 8.93 & 6.90 & 4.73 & 19.91 & 13.66 \\
\hline $5.0 \mathrm{M} \mathrm{NaDSO}_{4}$ & 14.77 & 10.11 & 6.35 & 4.35 & 21.12 & 14.45 \\
\hline
\end{tabular}

Table S2: Observed sulfate percentage product yields and water activity weighted mole fraction ratios for 1,2 -epoxybutane 


\begin{tabular}{|c|c|c|}
\hline & $\%$ yield & $\left(Y_{\text {sulfate }} / Y_{\text {water }}\right) * a_{\text {water }}$ \\
\hline \multicolumn{3}{|l|}{ Solution Set 1} \\
\hline $0.1 \mathrm{M} \mathrm{D}_{2} \mathrm{SO}_{4}$ & 1.36 & 0.76 \\
\hline $0.1 \mathrm{M} \mathrm{D}_{2} \mathrm{SO}_{4} 0.1 \mathrm{M} \mathrm{Na}_{2} \mathrm{SO}_{4}$ & 3.28 & 1.88 \\
\hline $0.1 \mathrm{M} \mathrm{D}_{2} \mathrm{SO}_{4} 0.2 \mathrm{M} \mathrm{Na}_{2} \mathrm{SO}_{4}$ & 5.35 & 3.15 \\
\hline $0.1 \mathrm{M} \mathrm{D}_{2} \mathrm{SO}_{4} 0.3 \mathrm{M} \mathrm{Na}_{2} \mathrm{SO}_{4}$ & 6.24 & 3.71 \\
\hline $0.1 \mathrm{M} \mathrm{D}_{2} \mathrm{SO}_{4} 0.4 \mathrm{M} \mathrm{Na}_{2} \mathrm{SO}_{4}$ & 8.26 & 5.03 \\
\hline $0.1 \mathrm{M} \mathrm{D}_{2} \mathrm{SO}_{4} 0.5 \mathrm{M} \mathrm{Na}_{2} \mathrm{SO}_{4}$ & 9.67 & 5.95 \\
\hline $0.1 \mathrm{M} \mathrm{D}_{2} \mathrm{SO}_{4} 0.7 \mathrm{M} \mathrm{Na}_{2} \mathrm{SO}_{4}$ & 11.76 & 7.49 \\
\hline $0.1 \mathrm{M} \mathrm{D}_{2} \mathrm{SO}_{4} 0.9 \mathrm{M} \mathrm{Na}_{2} \mathrm{SO}_{4}$ & 13.89 & 9.09 \\
\hline \multicolumn{3}{|l|}{ Solution Set 2} \\
\hline $0.2 \mathrm{M} \mathrm{NaDSO}_{4}$ & 3.12 & 1.79 \\
\hline $0.4 \mathrm{M} \mathrm{NaDSO}_{4}$ & 3.85 & 2.23 \\
\hline $0.7 \mathrm{M} \mathrm{NaDSO}_{4}$ & 5.34 & 3.15 \\
\hline $1.0 \mathrm{M} \mathrm{NaDSO}_{4}$ & 5.69 & 3.38 \\
\hline $1.5 \mathrm{M} \mathrm{NaDSO}_{4}$ & 6.82 & 4.10 \\
\hline $2.0 \mathrm{M} \mathrm{NaDSO}_{4}$ & 7.34 & 4.44 \\
\hline $3.0 \mathrm{M} \mathrm{NaDSO}_{4}$ & 8.78 & 5.36 \\
\hline $4.0 \mathrm{M} \mathrm{NaDSO}_{4}$ & 10.53 & 6.47 \\
\hline $5.0 \mathrm{M} \mathrm{NaDSO}_{4}$ & 12.44 & 7.67 \\
\hline
\end{tabular}

Table S3: Observed sulfate percentage product yields and water activity weighted mole fraction ratios for cis-2,3-epoxybutane 


\begin{tabular}{|l|c|c|}
\hline & $\%$ yield & $\left(\mathrm{Y}_{\text {sulfate }} / \mathrm{Y}_{\text {water }}\right)^{*} \mathrm{a}_{\text {water }}$ \\
\hline Solution Set 1 & 1.36 & 0.76 \\
\hline $0.1 \mathrm{M} \mathrm{D}_{2} \mathrm{SO}_{4}$ & 3.28 & 1.88 \\
\hline $0.1 \mathrm{M} \mathrm{D}_{2} \mathrm{SO}_{4} 0.1 \mathrm{M} \mathrm{Na}_{2} \mathrm{SO}_{4}$ & 5.35 & 3.15 \\
\hline $0.1 \mathrm{M} \mathrm{D}_{2} \mathrm{SO}_{4} 0.2 \mathrm{M} \mathrm{Na}_{2} \mathrm{SO}_{4}$ & 5.71 \\
\hline $0.1 \mathrm{M} \mathrm{D}_{2} \mathrm{SO}_{4} 0.3 \mathrm{M} \mathrm{Na}_{2} \mathrm{SO}_{4}$ & 6.24 & 5.03 \\
\hline $0.1 \mathrm{M} \mathrm{D}_{2} \mathrm{SO}_{4} 0.4 \mathrm{M} \mathrm{Na}_{2} \mathrm{SO}_{4}$ & 8.26 & 5.95 \\
\hline $0.1 \mathrm{M} \mathrm{D}_{2} \mathrm{SO}_{4} 0.5 \mathrm{M} \mathrm{Na}_{2} \mathrm{SO}_{4}$ & 9.67 & 7.49 \\
\hline $0.1 \mathrm{M} \mathrm{D}_{2} \mathrm{SO}_{4} 0.7 \mathrm{M} \mathrm{Na}_{2} \mathrm{SO}_{4}$ & 11.76 & 9.09 \\
\hline $0.1 \mathrm{M} \mathrm{D}_{2} \mathrm{SO}_{4} 0.9 \mathrm{M} \mathrm{Na}_{2} \mathrm{SO}_{4}$ & 13.89 & \\
\hline & & \\
\hline $\mathrm{Solution} \mathrm{Set} 2$ & 3.12 & 1.79 \\
\hline $0.2 \mathrm{M} \mathrm{NaDSO}_{4}$ & 3.85 & 2.23 \\
\hline $0.4 \mathrm{M} \mathrm{NaDSO}_{4}$ & 5.34 & 3.15 \\
\hline $0.7 \mathrm{M} \mathrm{NaDSO}_{4}$ & 5.69 & 3.38 \\
\hline $1.0 \mathrm{M} \mathrm{NaDSO}_{4}$ & 6.82 & 4.10 \\
\hline $1.5 \mathrm{M} \mathrm{NaDSO}_{4}$ & 7.34 & 4.44 \\
\hline $2.0 \mathrm{M} \mathrm{NaDSO}_{4}$ & 8.78 & 5.36 \\
\hline $3.0 \mathrm{M} \mathrm{NaDSO}_{4}$ & 10.53 & 6.47 \\
\hline $4.0 \mathrm{M} \mathrm{NaDSO}_{4}$ & 12.44 & 7.67 \\
\hline $5.0 \mathrm{M} \mathrm{NaDSO}_{4}$ & & \\
\hline
\end{tabular}

Table S4: Observed sulfate percentage product yields and water activity weighted mole fraction ratios for 2-methyl-1,2-epoxypropane 


\begin{tabular}{|c|c|c|c|c|c|}
\hline & \multicolumn{3}{|c|}{$1.0 \mathrm{M} \mathrm{SO}_{4}^{2-}$} & \multicolumn{2}{|c|}{$5.0 \mathrm{M} \mathrm{CH}_{3} \mathrm{OSO}_{3}^{-}$} \\
\hline & Y sulfate MS & Y sulfate LS & reference & Y sulfate MS & Y sulfate LS \\
\hline propylene oxide & 0.226 & 0.153 & & 0.020 & 0.014 \\
\hline glycidol & 0.087 & 0.331 & & 0.016 & 0.025 \\
\hline epifluorohydrin & 0.039 & 0.353 & & not observed & 0.047 \\
\hline epichlorohydrin & 0.014 & 0.367 & & not observed & 0.042 \\
\hline epibromohydrin & 0.023 & 0.357 & & not observed & 0.053 \\
\hline trans-2,3-epoxybutane & 0.079 & not applicable & 2 & not measured & not applicable \\
\hline cis-2,3-epoxybutane & 0.103 & not applicable & & not observed & not applicable \\
\hline 2,3-epoxy-1,4-butanediol & 0.303 & not applicable & & 0.096 & not applicable \\
\hline 1,2-epoxybutane & 0.110 & 0.110 & 2 & not observed & 0.012 \\
\hline 2-methyl-1,2-epoxypropane & 0.146 & not observed & 2 & not observed & not observed \\
\hline 2,3-dimethyl-2,3-epoxybutane & 0.120 & not applicable & 2 & not observed & not applicable \\
\hline trans- $\beta$-IEPOX & 0.200 & not observed & 3 & not observed & not observed \\
\hline
\end{tabular}

Table S5: Observed sulfate and methylsulfate mole fraction yields $\mathrm{s}^{2,3}$ 


\section{References}

1. $\quad$ Clegg, S. L.; Brimblecombe, P.; Exler, A. S., A thermodynamic model of the system $\mathrm{H}^{+}-$ $\mathrm{NH}_{4}{ }^{+}-\mathrm{SO}_{4}{ }^{2-}-\mathrm{NO}_{3}{ }^{-}-\mathrm{H}_{2} \mathrm{O}$ at tropospheric temperatures. J. Phys. Chem. A 1998, 102, 2137-2154.

2. Minerath, E. C.; Elrod, M. J., Assessing the potential for diol and hydroxy sulfate ester formation from the reaction of epoxides in tropospheric aerosols. Environ. Sci. Technol. 2009, 43, (5), 1386-1392.

3. Darer, A. I.; Cole-Filipiak, N. C.; O'Connor, A. E.; Elrod, M. J., Formation and stability of atmospherically relevant isoprene-derived organosulfates and organonitrates. Environ. Sci. Technol. 2011, 45, 1895-1902. 